\title{
Inverse Association between trans Isomeric and Long-Chain Polyunsaturated Fatty Acids in Pregnant Women and Their Newborns: Data from Three European Countries
}

\author{
T. Decsi $^{a} \quad$ C. Campoy ${ }^{b}$ H. Demmelmair ${ }^{d} \quad$ É. Szabó ${ }^{a} \quad$ T. Marosvölgyi ${ }^{a}$ \\ M. Escolanob $\quad$ G. Marchal ${ }^{b} \quad$ S. Krauss-Etschmann ${ }^{d} \quad$ M. Cruz ${ }^{c} \quad$ B. Koletzko ${ }^{d}$ \\ a University of Pécs, Department of Paediatrics, Pécs, Hungary; Departments of ${ }^{b}$ Paediatrics and ${ }^{c}$ Obstetrics and \\ Gynaecology, University of Granada, Granada, Spain; ' Dr. von Haunersches Kinderspital, University of Munich, \\ Munich, Germany
}

\section{Key Words}

Arachidonic acid · Docosahexaenoic acid $\cdot$ Neonate $\cdot$

Pregnancy $\cdot$ trans isomeric fatty acid

\begin{abstract}
Background: trans unsaturated fatty acids are thought to interfere with essential fatty acid metabolism. To extend our knowledge of this phenomenon, we investigated the relationship between trans isomeric and long-chain polyunsaturated fatty acids (LCPUFA) in mothers during pregnancy and in their infants at birth. Methods: Fatty acid composition of erythrocyte phosphatidylcholine (PC) and phosphatidylethanolamine (PE) was determined in Spanish $(n=120)$, German ( $n=78$ ) and Hungarian ( $n=43$ ) women at the 20th and 30th week of gestation, at delivery and in their newborns. $\boldsymbol{R} \boldsymbol{e}$ sults: At the 20th week of gestation, the sum of trans fatty acids in PE was significantly $(p<0.01)$ lower in Hungarian [0.73 (0.51), \% wt/wt, median (IQR)] than in Spanish [1.42 (1.36)] and German [1.30 (1.21)] women. Docosahexaenoic acid $(\mathrm{DHA})$ values in PE were significantly $(\mathrm{p}<0.01)$ higher in Hungarian [5.65 (2.09)] than in Spanish [4.37 (2.60)] or German [4.39 (3.3.2)] women. The sum of trans fatty acids significantly inversely correlated to DHA in PCs in Spanish $(r=$
\end{abstract}

\section{KARGER}

Fax +4161306 1234 E-Mail karger@karger.ch www.karger.com

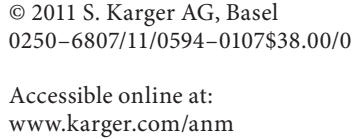

-0.37, $\mathrm{p}<0.001)$, German ( $\mathrm{n}=-0.77, \mathrm{p}<0.001$ ) and Hungarian $(r=-0.35, p<0.05)$ women, and in PEs in Spanish $(r=$ $-0.67, p<0.001)$ and German $(r=-0.71, p<0.001)$, but not in Hungarian $(r=-0.02)$ women. Significant inverse correlations were seen between trans fatty acids and DHA in PEs at the 30th week of gestation ( $n=241, r=-0.52$, $p<0.001$ ), at delivery $(n=241, r=-0.40, p<0.001)$ and in cord lipids $(n=$ $218, r=-0.28, p<0.001)$. Conclusion: Because humans cannot synthesize trans isomeric fatty acids, the data obtained in the present study support the concept that high maternal trans isomeric fatty acid intake may interfere with the availability of LCPUFA both for the mother and the fetus.

Copyright $\odot 2011$ S. Karger AG, Basel

\section{Introduction}

Docosahexaenoic acid (C22:6n-3, DHA) is an indispensable component of cell membranes and is incorporated in high concentrations into membrane phospholipids of brain and retina $[1,2]$. Randomized controlled trials found that enhanced availability of DHA during fetal $[3,4]$ or infantile [5] life may be associated with better outcome at tests investigating long-term cognitive and vi-

Dr. Tamás Decsi

Department of Paediatrics, University of Pécs

József A. u. 7

HU-7623 Pécs (Hungary)

Tel. +36 72535 900, E-Mail tamas.decsi@ aok.pte.hu 
sual development. Accumulating evidence suggests that the human fetus may benefit from an enhanced maternal dietary intake of DHA [for a recent review of randomized controlled trials, see 6]; it has recently been recommended that pregnant and lactating women should aim to achieve an average daily intake of at least $200 \mathrm{mg}$ DHA [7].

However, intakes of essential fatty acids and their longer-chain metabolites may not be the only dietary factors influencing the availability of long-chain polyunsaturated fatty acids (LCPUFAs) to the fetus. Data from a growing number of observational studies indicate that unsaturated fatty acids containing double bonds in trans stereoisomeric configuration may exert untoward effects not only on cardiovascular health in adults [for a recent review, see 8] but on essential fatty metabolism in the perinatal period as well. Significant inverse correlations were reported between trans isomeric fatty acids and LCPUFAs in cord blood lipids both in healthy full-term infants [9] and in full-term infants with an atopic trait [10], in cord vessel wall lipids in healthy full-term infants [11] as well as in plasma lipids in young preterm infants [12]. Similarly, an inverse association was found between trans isomeric fatty acids and LCPUFA in human milk both at the 6th week [13] and 6th month [14] of lactation. These data raise concerns about the nutritional adequacy of high maternal trans fatty acid intake in the perinatal period.

To extend our knowledge about the relationship between trans isomeric fatty acids and LCPUFA, we studied the fatty acid composition of erythrocyte membrane samples collected in a multicenter, randomized, doubleblind, placebo-controlled European study where pregnant women were supplemented either with a fish oil, with 5-methyltetrahydrofolic acid, or both, or with placebo from the 22nd week of gestation until delivery.

\section{Subjects and Methods}

\section{Study Design}

In this multicenter, randomized, double-blind, placebo-controlled supplementation study we investigated the fatty acid composition of erythrocyte membrane phosphatidylcholine (PC) and phosphatidylethanolamine (PE) in venous blood samples in sizeable groups of German, Hungarian and Spanish mothers at the 20th and 30th week of gestation and at delivery as well as in venous cord blood of their newborns. A detailed description of the study design, recruitment of subjects, dietary intervention, data collection, collection of biological material and power calculation was published elsewhere [15].
Briefly, apparently healthy pregnant women were recruited before the 20th week of gestation in the Departments of Obstetrics at the Ludwig Maximilians University, Munich, Germany, the University of Granada, Granada, Spain, and the University of Pécs, Pécs, Hungary. This study was conducted according to the guidelines laid down in the Declaration of Helsinki and all procedures involving human subjects were approved by the ethics committee of each participating center. After careful explanation of the study details, written informed consent was obtained from all subjects.

After inclusion in the study and before the 22nd week of gestation, participating women were randomly assigned to one of the four intervention groups. Each woman consumed daily one dose of the supplements providing either $500 \mathrm{mg}$ DHA and $150 \mathrm{mg}$ eicosapentaenoic acid (C20:5n-3, EPA) (Pronova Biocare, Lysaker, Norway) or $400 \mu \mathrm{g}$ 5-methyltetrahydrofolic acid (BASF, Ludwigshafen, Germany), or both, or placebo together with vitamins and minerals in amounts meeting the recommended intakes during the second half of pregnancy for European women. For the assessment of erythrocyte membrane PC and PE fatty acids $10 \mathrm{ml}$ maternal venous blood was collected into EDTA at study entry and again at the 30th week of gestation. At delivery, $12 \mathrm{ml}$ of maternal blood was collected into EDTA as well as $12 \mathrm{ml}$ of venous placental cord blood.

\section{Laboratory Methods}

For fatty acid analysis, the plasma was removed within $30 \mathrm{~min}$ and the erythrocyte mass was washed 3 times in isotonic sodium chloride solution. The last sediment was hemolyzed in $1 \mathrm{ml}$ volume of distilled water for $20 \mathrm{~min}$ at ambient temperature, and then $2 \mathrm{ml}$ ice-cold isopropylic alcohol with $0.5 \%$ butylated hydroxytoluolene (as antioxidant) was added drop-by-drop during continuous shaking. Thereafter, the samples were stored at $-80^{\circ} \mathrm{C}$ until further analysis. All samples were thawed only once when lipids were extracted by adding $3 \mathrm{ml}$ chloroform and the two internal standards (PE-heptadecanoate and PC-pentadecanoate esters dissolved in methanol). The mixture was shaken on vortex and the lower layer was aspirated and evaporated under nitrogen stream. The dry lipid extract was resolved in chloroform and added to silica gel plates (Merck 60, $10 \times 20 \mathrm{~cm}$ ). The mobile phase for the first run was hexane:diethylether:chloroform:acetic acid (21:6:3:1, vol/vol). The plate was dried under hood at ambient temperature and ran again with chloroform:methanol:water $65: 25: 4$, vol/vol). For positioning the PC and PE ester bands, the proper oleates were ran in parallel in every plate. The bands were stained with dichlorofluorescein, visualized under ultraviolet light and scrapped for transmethylation in $3 \mathrm{~N} \mathrm{HCl}$-methanol solution at $84^{\circ} \mathrm{C}$ for $40 \mathrm{~min}$.

Fatty acids were analyzed by high-resolution capillary gas-liquid chromatography (model 9001 gas chromatography; Finnigan/ Tremetrics, Austin, Tex., USA) with split injection, automatic sampler (A200SE, CTC Analytic, Switzerland) and flame ionization detector with a DB-23 cyanopropyl column of $60 \mathrm{~m}$ length (J\&W Scientific, Folsom, Calif., USA). Conditions during the analysis were as follows: temperature of injector at $80^{\circ} \mathrm{C}$ for 0.1 min, temperature increase by $180^{\circ} \mathrm{C}$ per min up to $280^{\circ} \mathrm{C}$, temperature of column area at $60^{\circ} \mathrm{C}$ for $0.2 \mathrm{~min}$, temperature increase by $40^{\circ} \mathrm{C}$ min up to $180^{\circ} \mathrm{C}$, a 5 -min hold period, temperature increase by $1.5^{\circ} \mathrm{C}$ per min up to $200^{\circ} \mathrm{C}$, an 8.5 -min hold period, temperature increase by $40^{\circ} \mathrm{C}$ per min up to $240^{\circ} \mathrm{C}$ and a $13-\mathrm{min}$ 
hold period. The constant linear velocity was $0.3 \mathrm{~m} / \mathrm{s}$ referred to $100^{\circ} \mathrm{C}$.

For identification of sample peaks we used two commercially available fatty acid methyl ester calibration mixtures (Supelco 37 FAME mix and NU-CHECK GLC reference 463) containing the fatty acids measured in the present study. These mixtures did not contain C22:5n-6, and since its precursor, C22:4n-6, coeluted with an unknown component, these two fatty acids were not included in our study. Fatty acid results were expressed as percentages (weight/weight) of fatty acids detected with a chain length between 14 and 24 carbon atoms. We detected three trans isomers in the PC fraction: trans hexadecenoic acid (C16:1t), trans octadecenoic acid (C18:1t) and trans octadecadienoic acid (C18: $2 \mathrm{n}-6 t t)$; the sum of these three trans isomers was entered into the correlation analysis as sum of the trans fatty acids. In the PE fraction, trans hexadecenoic acid coeluted with dimethyl acetal compounds; hence, we were able to quantify only C18 trans fatty acids.

\section{Statistical Methods}

We used SPSS for Windows, Release 15.0 (SPSS Inc., Chicago, Ill., USA) for the statistical analysis. The Kolmogorov-Smirnov test was used to evaluate the distribution of the data. Fatty acid compositional data among Spanish, German and Hungarian mothers were compared with the Kruskal-Wallis test. If this test indicated a significant variability of the data, the Bonferroni-corrected Mann-Whitney test was used to identify differences between populations.

Spearman rho correlation coefficients were calculated to investigate separately the relationship between trans isomeric fatty acids and LCPUFA at study entry, i.e. before intervention, for Spanish, German and Hungarian mothers. At later time points, i.e. during the intervention phase of the study, which means the 30 th week of gestation, delivery and cord blood, Pearson partial correlation analyses were carried out for the entire population after adjustment for the type of supplementation, nationality and normalization of data. Significance was set at $\mathrm{p}<0.05$ in all statistical analyses.

\section{Results}

Comparison of Fatty Acid Profiles among Populations at Study Entry

Here we only describe the differences seen in the principal fatty acids. Results of the statistical analyses for each and every fatty acid measured are shown in table 1 for PC and in table 2 for PE.

Values of linoleic acid (cis-9, cis-12-C18:2, LA) were significantly higher in Hungarian than in German and Spanish women in both PC and PE. Arachidonic acid (C20:4n-6, AA) values were significantly lower in German than in Hungarian and Spanish women in both PC and PE, and were significantly lower in Spanish than in Hungarian women in PE. $\alpha$-Linolenic acid (C18:3n-3, ALA) values were significantly lower in Spanish than in
German and Hungarian women in both PC and PE. EPA values were significantly lower in Spanish than in Hungarian women in PC and PE. In PC, DHA values were significantly higher in Spanish than in German women. In PE, DHA values were significantly higher in Hungarian than in German and Spanish women.

As to trans isomeric fatty acids, C16:1t values were significantly higher in German than in Hungarian women in PC. Values of C18:1t in PE were significantly lower in Hungarian than in Spanish or German women and were significantly lower in German than in Spanish women, whereas no differences were seen in PC. In both PC and $\mathrm{PE}, \mathrm{C} 18: 2 t t$ values were significantly lower in Hungarian than in Spanish or German women.

\section{Association of trans Isomeric Fatty Acids and \\ LCPUFA in Maternal Lipids at Study Entry}

C16:1t correlated significantly inversely with AA and DHA in PC in German mothers, whereas no correlation was seen in Spanish or Hungarian women. C18:1t correlated significantly inversely with ALA in PE in Spanish and German mothers (table 3). With the exception of DHA in PE in Hungarian women, statistically significant inverse correlations were seen between $\mathrm{C} 18: 1 t$ and both $\mathrm{AA}$ and DHA both in PC and PE in all the three populations. C18:2n-6tt correlated significantly inversely with LA in PC as well as with ALA in PE in Spanish women. Statistically significant inverse correlations were seen between C18:2n-6tt and AA in PC in German and Hungarian and in PE in German and Spanish women. C18: $2 n-6 t t$ correlated significantly inversely with DHA in both PC and PE in Spanish and German women. The sum of trans isomeric fatty acids significantly inversely correlated with ALA in PE in Spanish and German women. Almost uniformly significant inverse correlations were seen between the sum of trans isomeric fatty acids and AA and DHA; the only exception was the lack of correlation between the sum of trans fatty acids and DHA in $\mathrm{PE}$ in Hungarian women.

\section{Associations of trans Isomeric Fatty Acids and LCPUFA in Maternal and Infantile Phospholipids during the Study}

C16:1t correlated significantly positively with ALA in $\mathrm{PC}$ at the 30th week of gestation, whereas a significant inverse correlation was observed between C16:1t and AA at the 30th week of gestation (table 4). C18:1t correlated significantly inversely with LA in PE as well as with ALA in PE both at the 30th week of gestation and at delivery. Significant inverse correlations were seen between C18:1t 
Table 1. Fatty acid composition of erythrocyte membrane PC lipids at the 20th week of gestation in Spanish $(\mathrm{n}=120)$, German $(\mathrm{n}=78)$ and Hungarian $(\mathrm{n}=43)$ pregnant women

\begin{tabular}{|c|c|c|c|}
\hline & Spanish & German & Hungarian \\
\hline \multicolumn{4}{|l|}{ Saturated fatty acids } \\
\hline C14:0 & $0.39(0.25)^{\mathrm{A}, \mathrm{B}}$ & $0.63(0.33)^{\mathrm{A}}$ & $0.59(0.25)^{\mathrm{B}}$ \\
\hline $\mathrm{C} 16: 0$ & $33.19(7.79)^{\mathrm{A}, \mathrm{B}}$ & $38.72(9.20)^{\mathrm{A}}$ & $37.99(6.60)^{\mathrm{B}}$ \\
\hline C18:0 & $19.18(5.35)^{\mathrm{A}, \mathrm{B}}$ & $13.89(6.58)^{\mathrm{A}}$ & $13.15(3.70)^{\mathrm{B}}$ \\
\hline C20:0 & $0.31(0.25)^{\mathrm{a}, \mathrm{b}}$ & $0.24(0.24)^{\mathrm{a}}$ & $0.23(0.22)^{\mathrm{b}}$ \\
\hline $\mathrm{C} 22: 0$ & $0.26(0.28)$ & $0.24(0.14)$ & $0.23(0.22)$ \\
\hline C24:0 & $0.43(0.50)$ & $0.40(0.31)$ & $0.29(0.31)$ \\
\hline Sum of saturated & $53.82(4.60)$ & $54.76(4.12)^{\mathrm{a}}$ & $52.95(3.37)^{\mathrm{a}}$ \\
\hline \multicolumn{4}{|l|}{ cis monounsaturated fatty acids } \\
\hline $\mathrm{C} 16: 1 \mathrm{n}-7$ & $0.23(0.18)^{\mathrm{A}, \mathrm{B}}$ & $0.43(0.17)^{\mathrm{A}}$ & $0.44(0.19)^{\mathrm{B}}$ \\
\hline C18:1n-9 & $14.57(2.96)^{\mathrm{A}, \mathrm{B}}$ & $16.34(1.90)^{\mathrm{A}, \mathrm{C}}$ & $13.06(1.99)^{\mathrm{B}, \mathrm{C}}$ \\
\hline C18:1n-7 & $1.45(0.36)^{\mathrm{A}, \mathrm{a}}$ & $1.72(0.43)^{\mathrm{A}}$ & $1.61(0.37)^{\mathrm{a}}$ \\
\hline $\mathrm{C} 20: 1 \mathrm{n}-9$ & $0.33(0.22)^{\mathrm{a}}$ & $0.27(0.11)^{\mathrm{a}}$ & $0.27(0.15)$ \\
\hline $\mathrm{C} 22: 1 \mathrm{n}-9$ & $0.09(0.11)^{\mathrm{a}}$ & $0.06(0.07)^{\mathrm{A}}$ & $0.12(0.09)^{\mathrm{A}, \mathrm{a}}$ \\
\hline C24:1n-9 & $0.30(0.65)$ & $0.44(0.50)$ & $0.33(0.56)$ \\
\hline Sum of cis monounsaturated & $17.38(2.92)^{\mathrm{A}, \mathrm{B}}$ & $19.41(2.60)^{\mathrm{A}, \mathrm{C}}$ & $16.40(2.33)^{\mathrm{B}, \mathrm{C}}$ \\
\hline \multicolumn{4}{|l|}{ trans isomeric fatty acids } \\
\hline $\mathrm{C} 16: 1 t$ & $0.16(0.16)$ & $0.20(0.16)^{\mathrm{a}}$ & $0.14(0.12)^{\mathrm{a}}$ \\
\hline $\mathrm{C} 18: 1 t$ & $0.44(0.39)$ & $0.34(0.67)$ & $0.38(0.36)$ \\
\hline $\mathrm{C} 18: 2 \mathrm{n}-6 \mathrm{tt}$ & $0.18(0.15)^{\mathrm{a}}$ & $0.16(0.19)^{\mathrm{A}}$ & $0.10(0.12)^{\mathrm{A}, \mathrm{a}}$ \\
\hline Sum of trans & $0.82(0.53)$ & $0.79(0.90)$ & $0.68(0.43)$ \\
\hline \multicolumn{4}{|l|}{ n-6 polyunsaturated fatty acids } \\
\hline C18:2n-6 & $14.24(3.95)^{\mathrm{A}}$ & $15.64(3.26)^{\mathrm{B}}$ & $17.50(3.30)^{\mathrm{A}, \mathrm{B}}$ \\
\hline$C 18: 3 n-6$ & $0.03(0.09)^{\mathrm{A}}$ & $0.04(0.06)^{\mathrm{B}}$ & $0.11(0.15)^{\mathrm{A}, \mathrm{B}}$ \\
\hline$C 20: 2 n-6$ & $0.55(0.20)^{\mathrm{A}, \mathrm{a}}$ & $0.46(0.13)^{\mathrm{A}, \mathrm{B}}$ & $0.62(0.22)^{\mathrm{a}, \mathrm{B}}$ \\
\hline$C 20: 3 n-6$ & $1.79(0.69)^{\mathrm{A}}$ & $1.63(0.65)^{\mathrm{B}}$ & $2.27(0.64)^{\mathrm{A}, \mathrm{B}}$ \\
\hline$C 20: 4 n-6$ & $7.68(3.90)^{\mathrm{A}}$ & $3.99(3.62)^{\mathrm{A}, \mathrm{B}}$ & $6.59(3.22)^{\mathrm{B}}$ \\
\hline n-6 PUFA & $24.31(3.89)^{\mathrm{A}, \mathrm{a}}$ & $22.57(5.20)^{\mathrm{a}, \mathrm{B}}$ & $28.01(3.75)^{\mathrm{A}, \mathrm{B}}$ \\
\hline n-6 LCPUFA & $9.88(3.90)^{\mathrm{A}}$ & $6.24(3.87)^{\mathrm{A}, \mathrm{B}}$ & $9.84(3.97)^{\mathrm{B}}$ \\
\hline \multicolumn{4}{|l|}{ n-3 polyunsaturated fatty acids } \\
\hline C18:3n-3 & $0.08(0.07)^{\mathrm{A}, \mathrm{B}}$ & $0.16(0.10)^{\mathrm{A}}$ & $0.14(0.16)^{\mathrm{B}}$ \\
\hline$C 20: 3 n-3$ & $0.11(0.16)^{\mathrm{A}}$ & $0.06(0.09)^{\mathrm{B}}$ & $0.12(0.14)^{\mathrm{A}, \mathrm{B}}$ \\
\hline$C 20: 5 n-3$ & $0.09(0.08)^{\mathrm{A}}$ & $0.11(0.10)$ & $0.15(0.15)^{\mathrm{A}}$ \\
\hline$C 22: 5 n-3$ & $0.61(0.28)^{\mathrm{A}}$ & $0.40(0.68)$ & $0.36(0.40)^{\mathrm{A}}$ \\
\hline$C 22: 6 n-3$ & $2.29(1.55)^{\mathrm{A}}$ & $1.11(1.79)^{\mathrm{A}}$ & $1.84(1.73)$ \\
\hline n-3 PUFA & $3.57(1.81)^{\mathrm{A}}$ & $1.84(2.68)^{\mathrm{A}}$ & $2.68(2.41)$ \\
\hline n-3 LCPUFA & $3.43(1.78)^{\mathrm{A}, \mathrm{a}}$ & $1.66(2.63)^{\mathrm{A}}$ & $2.52(2.31)^{\mathrm{a}}$ \\
\hline
\end{tabular}

Data are $\%$ weight/weight expressed as medians (range from the 1 st to the 3 rd quartile). Values in rows with the same superscript are significantly different. Mann-Whitney test was performed if Kruskal-Wallis analysis of variance showed significant variability of data. ${ }^{\mathrm{a}, \mathrm{b}} \mathrm{p}<0.0167 ;{ }^{\mathrm{A}-\mathrm{C}} \mathrm{p}<0.001$.

and both AA and DHA in PE both at the 30th week of gestation and at delivery, whereas in PC significant inverse correlations were seen at the 30th week of gestation. No consistent results were seen when analyzing the association of C18:2n-6tt with LA and ALA. In contrast, C18:2n-6tt correlated significantly and inversely with
AA and DHA both at the 30th week of gestation and at delivery in PE and PC. Significant inverse correlations were seen between the sum of trans isomeric fatty acids and AA and DHA in maternal phospholipids; the only exception was the lack of correlation with DHA in PC at delivery. 
Table 2. Fatty acid composition of erythrocyte membrane PE lipids at the 20th week of gestation in Spanish $(\mathrm{n}=120)$, German $(\mathrm{n}=78)$ and Hungarian $(\mathrm{n}=43)$ pregnant women

\begin{tabular}{|c|c|c|c|}
\hline & Spanish & German & Hungarian \\
\hline \multicolumn{4}{|l|}{ Saturated fatty acids } \\
\hline $\mathrm{C} 14: 0$ & $0.26(0.20)^{\mathrm{a}, \mathrm{A}}$ & $0.32(0.18)^{\mathrm{a}}$ & $0.38(0.22)^{\mathrm{A}}$ \\
\hline $\mathrm{C} 16: 0$ & $24.24(3.80)^{\mathrm{a}}$ & $25.23(5.16)^{\mathrm{a}, \mathrm{A}}$ & $22.57(3.18)^{\mathrm{A}}$ \\
\hline C18:0 & $12.58(3.34)^{\mathrm{A}}$ & $12.25(3.23)^{\mathrm{B}}$ & $10.05(2.65)^{\mathrm{A}, \mathrm{B}}$ \\
\hline C20:0 & $0.37(0.85)^{\mathrm{a}}$ & $0.36(0.45)$ & $0.32(0.23)^{\mathrm{a}}$ \\
\hline $\mathrm{C} 22: 0$ & $0.44(0.92)^{\mathrm{A}}$ & $0.46(0.41)^{\mathrm{B}}$ & $0.26(0.26)^{\mathrm{A}, \mathrm{B}}$ \\
\hline C24:0 & $0.28(0.43)$ & $0.33(0.39)$ & $0.22(0.21)$ \\
\hline Sum of saturated & $39.30(7.29)^{\mathrm{A}}$ & $40.24(8.17)^{\mathrm{B}}$ & $33.94(5.31)^{\mathrm{A}, \mathrm{B}}$ \\
\hline \multicolumn{4}{|l|}{ cis monounsaturated fatty acids } \\
\hline $\mathrm{C} 16: 1 \mathrm{n}-7$ & $1.03(1.71)^{\mathrm{a}, \mathrm{A}}$ & $0.36(0.20)^{\mathrm{A}}$ & $0.43(0.31)^{\mathrm{a}}$ \\
\hline C18:1n-9 & $22.03(4.14)^{\mathrm{a}, \mathrm{A}}$ & $23.80(4.94)^{\mathrm{a}, \mathrm{B}}$ & $20.10(2.79)^{\mathrm{A}, \mathrm{B}}$ \\
\hline C18:1n-7 & $1.17(0.27)^{\mathrm{A}}$ & $1.22(0.26)$ & $1.31(0.38)^{\mathrm{A}}$ \\
\hline C20:1n-9 & $0.57(0.28)$ & $0.56(0.31)$ & $0.60(0.28)$ \\
\hline $\mathrm{C} 22: 1 \mathrm{n}-9$ & $0.14(0.13)$ & $0.12(0.13)$ & $0.16(0.20)$ \\
\hline $\mathrm{C} 24: 1 \mathrm{n}-9$ & $0.41(0.63)$ & $0.28(0.76)$ & $0.33(0.48)$ \\
\hline Sum of cis monounsaturated & $25.89(3.87)^{\mathrm{A}}$ & $26.73(4.60)^{\mathrm{B}}$ & $23.36(3.43)^{\mathrm{A}, \mathrm{B}}$ \\
\hline \multicolumn{4}{|l|}{ trans fatty acids } \\
\hline C18:1t & $1.19(1.18)^{\mathrm{a}, \mathrm{A}}$ & $1.07(1.13)^{\mathrm{a}, \mathrm{b}}$ & $0.68(0.44)^{\mathrm{b}, \mathrm{A}}$ \\
\hline $\mathrm{C} 18: 2 \mathrm{n}-6 \mathrm{tt}$ & $0.18(0.17)^{\mathrm{a}}$ & $0.19(0.13)^{\mathrm{A}}$ & $0.09(0.13)^{\mathrm{a}, \mathrm{A}}$ \\
\hline Sum of trans & $1.42(1.36)^{\mathrm{A}}$ & $1.30(1.21)^{\mathrm{a}}$ & $0.73(0.51)^{\mathrm{A}, \mathrm{a}}$ \\
\hline \multicolumn{4}{|l|}{ n-6 polyunsaturated fatty acids } \\
\hline $\mathrm{C} 18: 2 \mathrm{n}-6$ & $5.92(1.21)^{\mathrm{a}}$ & $5.72(1.33)^{\mathrm{A}}$ & $6.40(1.65)^{\mathrm{a}, \mathrm{A}}$ \\
\hline$C 18: 3 n-6$ & $0.02(0.20)^{\mathrm{a}, \mathrm{A}}$ & $0.08(0.10)^{\mathrm{a}}$ & $0.11(0.22)^{\mathrm{A}}$ \\
\hline$C 20: 2 n-6$ & $0.67(0.39)^{\mathrm{A}}$ & $0.57(0.25)^{\mathrm{A}, \mathrm{a}}$ & $0.69(0.30)^{\mathrm{a}}$ \\
\hline$C 20: 3 n-6$ & $1.18(0.44)^{\mathrm{a}}$ & $1.19(0.47)$ & $1.32(0.57)^{\mathrm{a}}$ \\
\hline$C 20: 4 n-6$ & $17.31(5.79)^{\mathrm{a}, \mathrm{A}}$ & $15.74(7.23)^{\mathrm{a}, \mathrm{B}}$ & $23.10(4.57)^{\mathrm{A}, \mathrm{B}}$ \\
\hline n-6 PUFA & $25.56(5.62)^{\mathrm{A}, \mathrm{a}}$ & $23.74(6.88)^{\mathrm{B}, \mathrm{a}}$ & $31.99(4.10)^{\mathrm{A}, \mathrm{B}}$ \\
\hline n-6 LCPUFA & $19.55(5.83)^{\mathrm{A}, \mathrm{a}}$ & $17.87(6.93)^{\mathrm{B}, \mathrm{a}}$ & $25.75(4.24)^{\mathrm{A}, \mathrm{B}}$ \\
\hline \multicolumn{4}{|l|}{ n-3 polyunsaturated fatty acids } \\
\hline $\mathrm{C} 18: 3 n-3$ & $0.12(0.07)^{\mathrm{A}, \mathrm{B}}$ & $0.19(0.14)^{\mathrm{A}}$ & $0.22(0.20)^{\mathrm{B}}$ \\
\hline$C 20: 3 n-3$ & $0.42(0.32)^{\mathrm{A}, \mathrm{a}}$ & $0.26(0.24)^{\mathrm{A}}$ & $0.24(0.35)^{\mathrm{a}}$ \\
\hline$C 20: 5 n-3$ & $0.26(0.24)^{\mathrm{a}}$ & $0.37(0.37)$ & $0.35(0.30)^{\mathrm{b}, \mathrm{a}}$ \\
\hline$C 22: 5 n-3$ & $1.59(0.74)^{\mathrm{A}, \mathrm{B}}$ & $2.11(1.50)^{\mathrm{A}}$ & $2.18(0.65)^{\mathrm{B}}$ \\
\hline$C 22: 6 n-3$ & $4.37(2.60)^{\mathrm{A}}$ & $4.39(3.32)^{\mathrm{a}}$ & $5.65(2.09)^{\mathrm{A}, \mathrm{a}}$ \\
\hline n-3 PUFA & $6.93(3.01)^{\mathrm{A}}$ & $7.41(5.00)$ & $9.03(2.62)^{\mathrm{A}}$ \\
\hline n-3 LCPUFA & $6.79(2.91)^{\mathrm{A}}$ & $7.27(4.84)$ & $8.79(2.80)^{\mathrm{A}}$ \\
\hline
\end{tabular}

Data are \% weight/weight expressed as medians (range from the 1st to the 3rd quartile). Values in rows with the same superscript are significantly different. The Bonferroni-corrected Mann-Whitney test was performed if Kruskal-Wallis analysis of variance showed significant variability of data. ${ }^{\mathrm{a}, \mathrm{b}} \mathrm{p}<0.0167 ;{ }^{\mathrm{A}, \mathrm{B}} \mathrm{p}<0.001$.

In cord blood, only one significant correlation was observed between trans isomeric fatty acids and the parent essential fatty acids LA and ALA. In contrast, all trans fatty acids showed a significant inverse correlation with both AA and DHA both in PC and PE in cord blood.
Associations of Product-to-Substrate Ratios of Polyunsaturated Fatty Acid Synthesis and trans Isomeric Fatty Acids in Maternal Lipids at Study

Entry

Product-to-substrate ratios for the delta-6-desaturase enzyme in $n-6$ polyunsaturated fatty acid synthesis (dihomo- $\gamma$-linolenic acid/LA) as well as various other prod- 
Table 3. Spearman correlation coefficients between polyunsaturated and trans isomeric fatty acids in erythrocyte membrane lipids in Spanish $(\mathrm{n}=120)$, German $(\mathrm{n}=78)$ and Hungarian $(\mathrm{n}=43)$ expecting women investigated at the 20th week of gestation

\begin{tabular}{|c|c|c|c|c|c|c|}
\hline \multirow[t]{2}{*}{ Fatty acid } & \multicolumn{3}{|l|}{ PC } & \multicolumn{3}{|l|}{$\mathrm{PE}$} \\
\hline & Spanish & German & Hungarian & Spanish & German & Hungarian \\
\hline \multicolumn{7}{|l|}{ Linoleic acid (C18:2n-6) } \\
\hline $\mathrm{C} 16: 1 \mathrm{n}-7 t$ & 0.03 & 0.13 & -0.05 & n.d. & n.d. & n.d. \\
\hline $\mathrm{C} 18: 1 \mathrm{n}-7 / 9 t$ & -0.11 & -0.16 & 0.06 & 0.05 & -0.03 & -0.06 \\
\hline $18: 2 n-6 t t$ & $-0.29^{b}$ & -0.06 & 0.19 & -0.01 & -0.10 & -0.01 \\
\hline Sum of trans isomeric & -0.17 & -0.12 & 0.11 & 0.05 & -0.03 & -0.05 \\
\hline \multicolumn{7}{|c|}{ Arachidonic acid (C20:4n-6) } \\
\hline $\mathrm{C} 16: 1 \mathrm{n}-7 t$ & 0.05 & $-0.36^{\mathrm{b}}$ & 0.11 & n.d. & n.d. & n.d. \\
\hline $\mathrm{C} 18: 1 \mathrm{n}-7 / 9 t$ & $-0.29^{\mathrm{b}}$ & $-0.71^{\mathrm{c}}$ & $-0.45^{\mathrm{b}}$ & $-0.45^{\mathrm{c}}$ & $-0.50^{c}$ & $-0.37^{\mathrm{a}}$ \\
\hline $\mathrm{C} 18: 2 \mathrm{n}-6 \mathrm{tt}$ & -0.15 & $-0.62^{c}$ & $-0.39^{\mathrm{a}}$ & $-0.29^{\mathrm{b}}$ & $-0.48^{\mathrm{c}}$ & -0.24 \\
\hline Sum of trans & $-0.28^{b}$ & $-0.77^{\mathrm{c}}$ & -0.47 & $-0.45^{\mathrm{c}}$ & $-0.51^{\mathrm{c}}$ & $-0.38^{\mathrm{a}}$ \\
\hline \multicolumn{7}{|c|}{ a-Linolenic acid (C18:3n-3) } \\
\hline $\mathrm{C} 16: 1 \mathrm{n}-7 t$ & 0.03 & 0.19 & 0.21 & n.d. & n.d. & n.d. \\
\hline $\mathrm{C} 18: 1 \mathrm{n}-7 / 9 t$ & -0.02 & 0.06 & 0.06 & $-0.26^{\mathrm{b}}$ & $-0.24^{\mathrm{a}}$ & -0.13 \\
\hline $\mathrm{C} 18: 2 \mathrm{n}-6 \mathrm{tt}$ & 0.05 & 0.22 & 0.20 & $-0.28^{\mathrm{b}}$ & -0.08 & -0.01 \\
\hline Sum of trans & 0.06 & 0.13 & 0.16 & $-0.26^{\mathrm{b}}$ & $-0.23^{\mathrm{a}}$ & -0.15 \\
\hline \multicolumn{7}{|l|}{$E P A(C 20: 5 n-3)$} \\
\hline $\mathrm{C} 16: 1 \mathrm{n}-7 t$ & $-0.33^{\mathrm{c}}$ & $-0.34^{\mathrm{a}}$ & 0.27 & n.d. & n.d. & n.d. \\
\hline $\mathrm{C} 18: 1 \mathrm{n}-7 / 9 t$ & -0.19 & $-0.46^{\mathrm{c}}$ & $-0.44^{\mathrm{a}}$ & $-0.37^{\mathrm{c}}$ & $-0.27^{\mathrm{a}}$ & -0.07 \\
\hline $\mathrm{C} 18: 2 \mathrm{n}-6 \mathrm{tt}$ & $-0.23^{\mathrm{a}}$ & $-0.38^{\mathrm{b}}$ & $-0.31^{\mathrm{a}}$ & $-0.47^{\mathrm{c}}$ & -0.09 & 0.07 \\
\hline Sum of trans & -0.13 & $-0.48^{\mathrm{c}}$ & $-0.39^{\mathrm{a}}$ & $-0.41^{\mathrm{c}}$ & $-0.25^{\mathrm{a}}$ & -0.01 \\
\hline \multicolumn{7}{|c|}{ Docosapentaenoic acid (C22:5n-3) } \\
\hline $\mathrm{C} 16: 1 \mathrm{n}-7 t$ & 0.11 & $-0.34^{\mathrm{a}}$ & 0.12 & n.d. & n.d. & n.d. \\
\hline $\mathrm{C} 18: 1 \mathrm{n}-7 / 9 t$ & $-0.33^{\mathrm{c}}$ & $-0.72^{\mathrm{c}}$ & $-0.31^{\mathrm{a}}$ & $-0.41^{\mathrm{c}}$ & $-0.68^{c}$ & 0.06 \\
\hline$C 18: 2 n-6 t t$ & -0.02 & $-0.69^{c}$ & $-0.40^{\mathrm{b}}$ & $-0.36^{c}$ & $-0.54^{\mathrm{c}}$ & -0.08 \\
\hline Sum of trans & $-0.24^{\mathrm{a}}$ & $-0.80^{c}$ & $-0.36^{\mathrm{a}}$ & $-0.42^{\mathrm{c}}$ & $-0.69^{c}$ & 0.05 \\
\hline \multicolumn{7}{|l|}{ DHA (C22:6n-3) } \\
\hline $\mathrm{C} 16: 1 \mathrm{n}-7 t$ & 0.18 & $-0.31^{\mathrm{a}}$ & 0.11 & n.d. & n.d. & n.d. \\
\hline $\mathrm{C} 18: 1 \mathrm{n}-7 / 9 t$ & $-0.43^{c}$ & $-0.73^{\mathrm{c}}$ & $-0.34^{\mathrm{a}}$ & $-0.68^{c}$ & $-0.69^{c}$ & -0.03 \\
\hline$C 18: 2 n-6 t t$ & $-0.22^{\mathrm{a}}$ & $-0.68^{\mathrm{c}}$ & -0.23 & $-0.50^{\mathrm{c}}$ & $-0.51^{\mathrm{c}}$ & -0.11 \\
\hline Sum of trans & $-0.37^{\mathrm{c}}$ & $-0.77^{\mathrm{c}}$ & $-0.35^{\mathrm{a}}$ & $-0.67^{c}$ & $-0.71^{\mathrm{c}}$ & -0.02 \\
\hline
\end{tabular}

Superscripts denote significant correlations: ${ }^{\mathrm{a}} \mathrm{p}<0.05 ;{ }^{\mathrm{b}} \mathrm{p}<0.01 ;{ }^{\mathrm{c}} \mathrm{p}<0.001$. n.d. $=$ C16:1 $t$ not detected.

uct-to-substrate ratios characterizing synthetic pathway activity are shown in table 5. All statistically significant associations indicated inverse relations between trans isomeric fatty acids and the activity of LCPUFA synthesis.

\section{Discussion}

The present study is an analytical extension of a randomized, controlled dietary supplementation trial carried out in pregnant women living in three European countries presumably with a different amount of DHA in their natural diet. The assumption of different natural dietary DHA intakes in the three study populations was confirmed later on by the analysis of food frequency questionnaire data recorded during the study [16]. Either expressed in absolute amounts or adjusted for energy intakes, the amount of DHA was significantly higher in the diet of Spanish than in the diet of German or Hungarian mothers. Absolute amounts of DHA intakes were also significantly higher in Hungarian than in German mothers [16]; however, this difference disappeared following adjustment for energy intakes. 
Table 4. Partial correlation coefficients between trans fatty acids and the principal n-3 and n-6 polyunsaturated fatty acids during pregnancy and in cord blood samples

\begin{tabular}{|c|c|c|c|}
\hline & $\begin{array}{l}\text { 30th week } \\
(\mathrm{n}=241)\end{array}$ & $\begin{array}{l}\text { Delivery } \\
(\mathrm{n}=241)\end{array}$ & $\begin{array}{l}\text { Cord blood } \\
(\mathrm{n}=218)\end{array}$ \\
\hline \multicolumn{4}{|l|}{$P C$} \\
\hline \multicolumn{4}{|l|}{$C 18: 2 n-6$} \\
\hline $\mathrm{C} 16: 1 t$ & +0.02 & -0.02 & +0.02 \\
\hline C18:1t & $-0.27^{\mathrm{c}}$ & $-0.26^{c}$ & -0.05 \\
\hline $\mathrm{C} 18: 2 \mathrm{n}-6 t t$ & $-0.28^{\mathrm{c}}$ & $-0.19^{\mathrm{a}}$ & -0.08 \\
\hline Sum of trans & $-0.24^{\mathrm{c}}$ & $-0.24^{\mathrm{b}}$ & -0.03 \\
\hline \multicolumn{4}{|l|}{ C20:4n-6 } \\
\hline C16:1t & $-0.30^{\mathrm{c}}$ & -0.13 & $-0.32^{\mathrm{c}}$ \\
\hline $\mathrm{C} 18: 1 t$ & $-0.29^{c}$ & -0.04 & $-0.32^{\mathrm{c}}$ \\
\hline $\mathrm{C} 18: 2 \mathrm{n}-6 \mathrm{tt}$ & $-0.25^{c}$ & $-0.16^{\mathrm{a}}$ & $-0.40^{\mathrm{c}}$ \\
\hline Sum of trans & $-0.40^{\mathrm{c}}$ & $-0.16^{\mathrm{a}}$ & $-0.49^{c}$ \\
\hline \multicolumn{4}{|l|}{$\mathrm{C} 18: 3 n-3$} \\
\hline $\mathrm{C} 16: 1 t$ & $+0.18^{\mathrm{a}}$ & +0.12 & +0.10 \\
\hline $\mathrm{C} 18: 1 t$ & -0.10 & -0.03 & -0.03 \\
\hline$C 18: 2 n-6 t t$ & $-0.18^{\mathrm{b}}$ & -0.04 & -0.09 \\
\hline Sum of trans & -0.06 & +0.01 & -0.01 \\
\hline \multicolumn{4}{|l|}{$C 22: 6 n-3$} \\
\hline $\mathrm{C} 16: 1 t$ & -0.13 & -0.04 & $-0.27^{\mathrm{c}}$ \\
\hline $\mathrm{C} 18: 1 t$ & $-0.29^{c}$ & -0.13 & $-0.29^{c}$ \\
\hline $\mathrm{C} 18: 2 \mathrm{n}-6 \mathrm{tt}$ & $-0.20^{\mathrm{b}}$ & -0.12 & $-0.22^{\mathrm{b}}$ \\
\hline Sum of trans & $-0.35^{\mathrm{c}}$ & -0.19 & $-0.40^{\mathrm{c}}$ \\
\hline \multicolumn{4}{|l|}{$P E$} \\
\hline \multicolumn{4}{|l|}{$C 18: 2 n-6$} \\
\hline C18:1t & -0.08 & -0.10 & $0.17^{\mathrm{a}}$ \\
\hline $\mathrm{C} 18: 2 \mathrm{n}-6 t t$ & $+0.14^{\mathrm{a}}$ & +0.05 & -0.07 \\
\hline Sum of trans & -0.05 & -0.08 & 0.17 \\
\hline \multicolumn{4}{|l|}{$C 20: 4 n-6$} \\
\hline C18:1t & $-0.47^{\mathrm{c}}$ & $-0.24^{\mathrm{c}}$ & $-0.38^{\mathrm{c}}$ \\
\hline $\mathrm{C} 18: 2 \mathrm{n}-6 \mathrm{tt}$ & $-0.42^{\mathrm{c}}$ & $-0.34^{\mathrm{c}}$ & $-0.24^{\mathrm{b}}$ \\
\hline Sum of trans & $-0.52^{\mathrm{c}}$ & $-0.27^{\mathrm{c}}$ & $-0.43^{\mathrm{c}}$ \\
\hline \multicolumn{4}{|l|}{$C 18: 3 n-3$} \\
\hline C18:1t & $-0.27^{\mathrm{c}}$ & $-0.25^{\mathrm{c}}$ & -0.13 \\
\hline $\mathrm{C} 18: 2 \mathrm{n}-6 t t$ & -0.09 & $-0.25^{\mathrm{c}}$ & +0.12 \\
\hline Sum of trans & $-0.26^{\mathrm{c}}$ & $-0.27^{\mathrm{c}}$ & -0.08 \\
\hline \multicolumn{4}{|l|}{$C 22: 6 n-3$} \\
\hline C18:19t & $-0.50^{c}$ & $-0.40^{c}$ & $-0.22 b$ \\
\hline $\mathrm{C} 18: 2 \mathrm{n}-6 t t$ & $-0.35^{\mathrm{c}}$ & $-0.20^{\mathrm{b}}$ & $-0.28^{c}$ \\
\hline Sum of trans & $-0.52^{\mathrm{c}}$ & $-0.40^{\mathrm{c}}$ & $-0.28^{\mathrm{c}}$ \\
\hline
\end{tabular}

Correlations were corrected for nationality and supplementation and were normalized before evaluating Pearson's partial correlation. ${ }^{\mathrm{a}} \mathrm{p}<0.05 ;{ }^{\mathrm{b}} \mathrm{p}<0.01 ;{ }^{\mathrm{c}} \mathrm{p}<0.001$.

The significantly higher contribution of DHA to PC seen in Spanish compared to German or Hungarian mothers at study entry can easily be explained on the basis of the differences seen in DHA intake. Indeed, statistically significant positive correlations were reported be- tween dietary DHA intake and erythrocyte membrane DHA values both in small $(\mathrm{n}=35)$ and sizeable $(\mathrm{n}=1,550)$ cohorts of pregnant women $[17,18]$. However, the significantly higher contribution of DHA to PE in Hungarian compared to Spanish or German mothers can only partly be explained by dietary DHA intakes, namely, that the diet of Hungarian mothers contained significantly higher absolute amounts of DHA than the diet of German mothers. In contrast, dietary DHA intakes cannot explain the difference seen between Spanish and Hungarian mothers in PE. Moreover, there were entirely contradictory results when DHA values were compared between Spanish and Hungarian mothers either in PC or in $\mathrm{PE}$, i.e. significantly lower DHA in PC, but significantly higher DHA in PE in Hungarian compared to Spanish mothers.

One potential explanation for the apparent controversy in DHA concentrations in PC between Spanish and Hungarian women at study entry may be related to trans isomeric fatty acids showing a different picture in PC and PE: while the contribution of trans isomeric fatty acids to the fatty acid composition of PC did not differ between Spanish and Hungarian mothers, in PE Spanish mothers exhibited a significantly higher contribution of C18 trans isomers than Hungarian women (higher by $80 \%$ in C18:1t and by nearly $50 \%$ in C18:2n-6tt). We are unaware of data on differences in the incorporation of trans isomeric fatty acids into erythrocyte membrane PC or PE. However, recently Harvey et al. [19] compared the incorporation of LA and C18:2n-6tt into phospholipids, triacylglycerols and cholesteryl esters in human aortic endothelial cells. More trans-9,trans-12-C18:2 were enriched in phospholipids than cis-9,cis-12-C18:2, whereas similar levels of cis-9,cis-12-C18:2 and trans-9,trans-12C18:2 enrichment were observed in triacylglycerols, and no enrichment was seen in cholesteryl esters [19]. These data suggest that the enrichment of trans isomeric fatty acids may be different between PC and PE as well, as we actually observed in the present study, and this raises the possibility that the different availability of trans isomeric fatty acids in the membrane may lead to further consequences for membrane metabolism.

trans isomeric fatty acids may interfere either with the availability of DHA for the synthesis of membrane structures or with the local incorporation of preformed DHA molecules. As far as the potential influence of trans fatty acids on DHA synthesis is concerned, it has been demonstrated that feeding pregnant sows with high doses of trans isomeric fatty acids resulted in a significant decrease of DHA to aortic tissue phospholipids of piglets investi- 
Table 5. Spearman correlation coefficients between product-to-substrate ratios of polyunsaturated fatty acid synthesis and trans isomeric fatty acids in erythrocyte membrane lipids in Spanish $(\mathrm{n}=120)$, German $(\mathrm{n}=78)$ and Hungarian $(\mathrm{n}=43)$ expecting mothers investigated at the 20 th week of gestation

\begin{tabular}{|c|c|c|c|c|c|c|}
\hline \multirow[t]{2}{*}{ Fatty acid } & \multicolumn{3}{|l|}{ PC } & \multicolumn{3}{|l|}{$\mathrm{PE}$} \\
\hline & Spanish & German & Hungarian & Spanish & German & Hungarian \\
\hline \multicolumn{7}{|l|}{ DHGLA/LA } \\
\hline $\mathrm{C} 16: 1 \mathrm{n}-7 t$ & -0.20 & -0.19 & 0.10 & n.d. & n.d. & n.d. \\
\hline $\mathrm{C} 18: 1 \mathrm{n}-7 / 9 t$ & -0.004 & $-0.44^{\mathrm{c}}$ & -0.20 & $-0.37^{\mathrm{c}}$ & $-0.49^{c}$ & 0.24 \\
\hline $\mathrm{C} 18: 2 \mathrm{n}-6 \mathrm{tt}$ & 0.07 & $-0.31^{\mathrm{a}}$ & -0.16 & -0.17 & $-0.39^{c}$ & 0.08 \\
\hline Sum of trans isomeric & -0.001 & $-0.46^{\mathrm{c}}$ & -0.21 & $-0.36^{\mathrm{c}}$ & $-0.50^{c}$ & 0.25 \\
\hline \multicolumn{7}{|l|}{$A A / D H G L A$} \\
\hline $\mathrm{C} 16: 1 \mathrm{n}-7 t$ & 0.002 & $-0.42^{\mathrm{b}}$ & 0.07 & n.d. & n.d. & n.d. \\
\hline C18:1n-7/9t & $-0.24^{\mathrm{a}}$ & $-0.53^{\mathrm{c}}$ & $-0.32^{\mathrm{b}}$ & -0.13 & -0.07 & -0.30 \\
\hline $\mathrm{C} 18: 2 \mathrm{n}-6 \mathrm{tt}$ & -0.08 & $-0.59^{c}$ & $-0.39^{\mathrm{b}}$ & -0.11 & -0.04 & -0.14 \\
\hline Sum of trans & $-0.21^{\mathrm{a}}$ & $-0.62^{\mathrm{c}}$ & $-0.36^{\mathrm{a}}$ & -0.13 & -0.07 & $-0.31^{\mathrm{c}}$ \\
\hline \multicolumn{7}{|l|}{$E P A / A L A$} \\
\hline $\mathrm{C} 16: 1 \mathrm{n}-7 t$ & $0.24^{\mathrm{a}}$ & $-0.34^{\mathrm{a}}$ & 0.08 & n.d. & n.d. & n.d. \\
\hline C18:1n-7/9t & -0.13 & $-0.53^{\mathrm{c}}$ & $-0.45^{\mathrm{b}}$ & -0.02 & -0.01 & 0.08 \\
\hline $\mathrm{C} 18: 2 \mathrm{n}-6 \mathrm{tt}$ & -0.19 & $-0.55^{\mathrm{c}}$ & $-0.45^{\mathrm{b}}$ & -0.05 & 0.06 & 0.09 \\
\hline Sum of trans & -0.13 & $-0.58^{\mathrm{c}}$ & $-0.48^{\mathrm{b}}$ & -0.04 & 0.01 & 0.15 \\
\hline \multicolumn{7}{|l|}{$D P A / E P A$} \\
\hline $\mathrm{C} 16: 1 \mathrm{n}-7 t$ & $-0.25^{\mathrm{a}}$ & -0.01 & -0.05 & n.d. & n.d. & n.d. \\
\hline $\mathrm{C} 18: 1 \mathrm{n}-7 / 9 t$ & -0.21 & $-0.35^{\mathrm{b}}$ & 0.08 & 0.16 & $-0.23^{\mathrm{a}}$ & 0.11 \\
\hline $\mathrm{C} 18: 2 \mathrm{n}-6 \mathrm{tt}$ & $-0.23^{\mathrm{a}}$ & $-0.41^{\mathrm{b}}$ & -0.04 & $-0.30^{\mathrm{b}}$ & $-0.39^{c}$ & 0.03 \\
\hline Sum of trans & -0.02 & $-0.37^{b}$ & 0.02 & 0.19 & $-0.25^{\mathrm{a}}$ & 0.07 \\
\hline \multicolumn{7}{|l|}{$D H A / D P A$} \\
\hline $\mathrm{C} 16: 1 \mathrm{n}-7 t$ & 0.13 & 0.07 & -0.07 & n.d. & n.d. & n.d. \\
\hline $\mathrm{C} 18: 1 \mathrm{n}-7 / 9 t$ & -0.17 & 0.11 & 0.10 & $-0.42^{\mathrm{c}}$ & -0.12 & -0.20 \\
\hline $\mathrm{C} 18: 2 \mathrm{n}-6 \mathrm{tt}$ & $-0.36^{\mathrm{c}}$ & 0.24 & $-0.32^{\mathrm{a}}$ & $-0.30^{\mathrm{b}}$ & -0.03 & -0.20 \\
\hline Sum of trans & $-0.21^{\mathrm{a}}$ & 0.16 & 0.14 & $-0.40^{\mathrm{c}}$ & -0.13 & -0.19 \\
\hline
\end{tabular}

Superscripts denote significant correlations: ${ }^{\mathrm{a}} \mathrm{p}<0.05 ;{ }^{\mathrm{b}} \mathrm{p}<0.01 ;{ }^{\mathrm{c}} \mathrm{p}<0.001$. DHGLA = Dihomo- $\gamma$-linolenic acid $(\mathrm{C} 20: 3 \mathrm{n}-6)$; DPA = docosapentaenoic acid (C22:5n-3); n.d. = not detected.

gated at the age of 3 days [20]. This finding was in concert with the effect of trans isomeric fatty acids to inhibit delta6-desaturase both in vitro [21] and in rat liver microsomes [22], and was interpreted as the effect of hydrogenated fats to inhibit LCPUFA synthesis [20]. It should also be noted that in the present study trans isomeric fatty acids were significantly inversely related to the parent $\mathrm{n}-3$ LCPUFA, ALA. This inverse relationship is usually not seen in other studies relating trans fatty acids to LCPUFA, and may possibly reflect the lower trans fatty acid content of margarines and spreads with higher ALA contents.

Incorporation of DHA into membrane lipids is seen as a complex process consisting of partitioning of dissolved DHA monomers into the membrane, transfer of monomers from aggregates absorbed onto the membrane and fusion of aggregates with the membrane [23]. The ratelimiting step of DHA incorporation appears to be the dissociation or desorption of the molecule from the donor system. It has been demonstrated that the increase of molecular length of the fatty acids in the donor medium by two $\mathrm{CH}_{2}$ groups increases the half-times for the transfer by a factor of about 10 [24]. Furthermore, it has been demonstrated that various membrane properties are also modified by the presence of trans as compared to cis isomeric fatty acids [25]. On the basis of these considerations it seems to be plausible to assume that the presence of trans double bonds may also affect DHA incorporation into PC and PE. Such an effect may explain, at least partly, the apparent controversy seen in comparing DHA between Spanish and Hungarian women in PC or PE. 
As demonstrated in table 3, all relationships between trans fatty acid concentrations and the $n-6$ and $n-3$ fatty acid levels studied were negative, and most of them were statistically significant as well. It is to be noted that trans isomeric fatty acids were significantly and inversely related to the intermediary metabolites of DHA synthesis, i.e. to EPA and docosapentaenoic acid (C22:5n-3) as well. Significance was less prominent for the Hungarians, however, which may be due to a power problem. Although the Hungarian group size seems acceptable, trans fatty acid levels - and 'trans exposure ranges' in particular appear generally lower in the Hungarians than in the other groups. As a result, a significant trans LCPUFA relationship may require a higher number of cases in the Hungarian group than in the other groups.

The significant inverse associations seen between trans isomeric fatty acids and DHA at study entry support further the concept of modifying effect of trans fatty acids on DHA status. It would be tempting to interpret the significant and inverse relations between some trans fatty acids and various product-to-substrate ratios in LCPUFA synthesis as indicators of disturbed conversion of essential fatty acids to their longer-chain metabolites. However, the biochemical meaning of product-to-substrate ratios in essential fatty acid metabolism has not yet been firmly established for plasma lipid classes, and even less is known about the potential meaning of such ratios in erythrocyte membrane lipids. Therefore, caution is needed in interpreting the apparently impressive data shown in table 5.

To the best of our knowledge, the present study is the first to provide a detailed description of an inverse association between trans isomeric fatty acids and LCPUFA in blood samples of pregnant women, although comparable results have been briefly mentioned by others [26, 27]. Moreover, enhancement of the average dietary DHA intake of the population (i.e. the supplementation used in half of the women participating in the present study) did not make the inverse association between trans fatty acids and DHA disappear by the 30th week of gestation or by delivery. It is to be noted that the contribution of trans fatty acids to erythrocyte membrane lipids in the present study was well in the range usually reported for healthy adults: $1-2 \%$ for $\mathrm{C} 18: 1 t$ and $0.25-0.4 \%$ for $\mathrm{C} 18: 2 \mathrm{n}-6 \mathrm{tt}$ [28-30]. Hence, the effect of trans fatty acids in interfering with the availability of LCPUFA seems to manifest itself also at moderate trans fatty acid and enhanced DHA intakes.

The inverse association of trans isomeric fatty acids with AA and DHA in venous cord blood lipids seen in the present study is in concert with previous reports on inverse association between trans isomeric fatty acids and LCPUFA in venous cord blood plasma lipids in Austrian newborns [10], in cord blood vessel wall lipids in Dutch neonates [11] and in venous blood plasma lipids in 4-dayold German preterm infants [12]. Higher intrauterine exposure to trans fatty acids was related to untoward functional outcomes in more than one study. trans Fatty acids in umbilical cord blood vessel wall lipid showed a negative association with the so-called neurologic optimality score investigated in a group of 317 toddlers at the age of 18 months [31]. Moreover, trans fatty acids in maternal plasma lipids inversely correlated with birth weight and gestational age in a study on 44 Polish women [32], and percent calories from trans fatty acids were positively and independently associated with fetal loss in a recent survey on 104 American women participating in the Princeton School cardiovascular risk study [33]. Though no association between birth dimensions and maternal phospholipid C18:1t values were reported in a very recent study on 782 Dutch mother-infant pairs [34], the question remains open whether trans fatty acids may affect obstetric pregnancy outcomes [33,35].

In summary, we report inverse associations between trans isomeric fatty acids and DHA in a sizeable group of European women investigated at three phases of pregnancy. Though these observational data do not allow a direct conclusion to be drawn on functional consequences of dietary trans fatty acid exposure to pregnant women, the results reported certainly raise further concerns about the nutritional adequacy of high trans fatty acid intakes during pregnancy.

\section{Acknowledgements}

The studies reported herein have been carried out with partial financial support from the Commission of the European Communities, the specific RTD Programme 'Quality of Life and Management of Living Resources', within the 5th Framework Programme [contract No. QLK1-CT-1999-00888 NUHEAL (Nutraceuticals for a healthier life)] and 7th Framework Programme [grant agreement No. 212652 NUTRIMENTHE (The Effect of Diet on the Mental Performance of Children)]. This manuscript does not necessarily reflect the views of the Commission and in no way anticipates any future policy in this area.

\section{Disclosure Statement}

None of the authors had any conflict of interest in connection with this study. 


\section{References}

1 Farquharson J, Jamieson EC, Abbasi KA, Patrick WJ, Logan RW, Cockburn F: Effect of diet on the fatty acid composition of the major phospholipids of infant cerebral cortex. Arch Dis Child 1995;72:198-203.

2 Makrides M, Neumann MA, Byard RW, Simmer K, Gibson RA: Fatty acid composition of brain, retina, and erythrocytes in breast- and formula-fed infants. Am J Clin Nutr 1994;60:189-194.

3 Helland IB, Smith L, Saarem K, Saugstad OD, Drevon CA: Maternal supplementation with very-long-chain $n-3$ fatty acids during pregnancy and lactation augments children's IQ at 4 years of age. Pediatrics 2003;111:e39-e44.

4 Bakker EC, Hornstra G, Blanco CE, Vles JSH: Relationship between long-chain polyunsaturated fatty acids at birth and motor function at 7 years of age. Eur J Clin Nutr 2009;63:499-504.

5 Birch EE, Garfield S, Castaneda Y, Hughbanks-Wheaton D, Uauy R, Hoffman D: Visual acuity and cognitive outcomes at 4 years of age in a double-blind, randomized trial of long-chain polyunsaturated fatty acid-supplemented infant formula. Early Hum Dev 2007;83:279-284.

6 Decsi T: Effects of supplementing LCPUFA to the diet of pregnant women: data from RCT. Adv Exp Med Biol 2009;646:65-69.

7 Koletzko B, Lien E, Agostoni C, Bohles H, Campoy C, Cetin I, Decsi T, Dudenhausen JW, Dupont C, Forsyth S, Hoesli I, Holzgreve W, Lapillonne A, Putet G, Secher NJ, Symonds M, Szajewska H, Willatts P, Uauy R: The roles of long-chain polyunsaturated fatty acids in pregnancy, lactation and infancy: review of current knowledge and consensus recommendations. J Perinat Med 2008;36: 5-14.

8 Mozaffarian D, Aro A, Willett WC: Health effects of trans-fatty acids: experimental and observational evidence. Eur J Clin Nutr 2009;63:S5-S21.

9 Elias SL, Innis SM: Infant plasma trans, n-6, and $\mathrm{n}-3$ fatty acids and conjugated linoleic acids are related to maternal plasma fatty acids, length of gestation, and birth weight and length. Am J Clin Nutr 2001;73:807-814.

10 Decsi T, Burus I, Molnár S, Minda H, Veitl V: Inverse association between trans isomeric and long-chain polyunsaturated fatty acids in cord blood lipids of full-term infants. Am J Clin Nutr 2001;74:364-368.

11 Decsi T, Boehm G, Tjoonk HM, Molnár S, Dijck-Brouwer DA, Hadders-Algra M, Martini I, Muskiet F, Boersma R: Trans isomeric octadecenoic acids are related inversely to arachidonic acid and DHA and positively related to mead acid in umbilical vessel wall lipids. Lipids 2002;37:959-965.

12 Koletzko B: trans fatty acids may impair biosynthesis of long-chain polyunsaturates and growth in man. Acta Paediatr 1992;81:302306.
13 Szabó É, Boehm G, Beermann C, Weyermann M, Brenner $\mathrm{H}$, Rothenbacher D, Decsi T: trans octadecenoic acid and trans octadecadienoic acid are inversely related to long-chain polyunsaturates in human milk: results of a large birth cohort study. Am J Clin Nutr 2007;85:1320-1326.

14 Szabó É, Boehm G, Beermann C, Weyermann M, Brenner H, Rothenbacher D, Decsi T: Fatty acid profile comparisons in human milk sampled from the same mothers at the sixth week and the sixth month of lactation. J Pediatr Gastroenterol Nutr 2010;50:316320.

15 Krauss-Etschmann S, Shadid R, Campoy C, Hoster E, Demmelmair H, Jimenez M, Gil A, Rivero M, Veszprémi B, Decsi T, Koletzko B: Effects of fish-oil and folate supplementation of pregnant women on maternal and fetal plasma concentrations of docosahexaenoic acid and eicosapentaenoic acid: a European randomized multicenter trial. Am J Clin Nutr 2007;85:1392-1400.

16 Franke C, Verwied-Jorky S, Campoy C, Trak-Fellermeier M, Decsi T, Dolz V, Koletzko B: Dietary intake of natural sources of docosahexaenoic acid and folate in pregnant women of three European cohorts. Ann Nutr Metab 2008;53:167-174.

17 Parra MS, Schnaas L, Meydani M, Perroni E, Martinez S, Romieu I: Erythrocyte cell membrane phospholipid levels compared against reported dietary intakes of polyunsaturated fatty acids in pregnant Mexican women. Public Health Nutr 2002;5:931-937.

18 Donahue SM, Rifas-Shiman SL, Olsen SF, Gold DR, Gillman MW, Oken E: Associations of maternal prenatal dietary intake of n-3 and n-6 fatty acids with maternal and umbilical cord blood levels. Prostaglandins Leukot Essent Fatty Acids 2009;80:289-296.

19 Harvey KA, Arnold T, Rasool T, Antalis C, Miller SJ, Siddiqui RA: trans-fatty acids induce pro-inflammatory responses and endothelial cell dysfunction. Br J Nutr 2008;99: 723-731.

20 Kummerow FA, Zhou Q, Mahfouz MM, Smiricky MR, Grieshop CM, Schaeffer DJ: trans fatty acids in hydrogenated fat inhibited the synthesis of the polyunsaturated fatty acids in the phospholipid of arterial cells. Life Sci 2004; 74:2707-2723.

21 Mahfouz MM, Johnson S, Holman RT: The effect of isomeric trans-18:1 acids on the desaturation of palmitic, linoleic and eicosa8,11,14-trienoic acids by rat liver microsomes. Lipids 1980;15:100-107.

22 Mahfouz MM, Smith TL, Kummerow FA Effect of dietary fats on desaturase activities and the biosynthesis of fatty acids in rat-liver microsomes. Lipids 1984;19:214-222.

23 Chen IA, Szostak JW: A kinetic study of the growth of fatty acid vesicles. Biophys J 2004; 87:988-998.
24 Thomas RM, Baici A, Werder M, Schulthess G, Hauser H: Kinetics and mechanism of long-chain fatty acid transport into phosphatidylcholine vesicles from various donor systems. Biochemistry 2002;41:1591-1601.

25 Roach C, Feller SE, Ward JA, Shaikh SR, Zerouga $\mathrm{M}$, Stillwell W: Comparison of cis and trans fatty acid containing phosphatidylcholines on membrane properties. Biochemistry 2004;43:6344-6351.

26 Dirix CE, Kester AD, Hornstra G: Associations between neonatal birth dimensions and maternal essential and trans fatty acid contents during pregnancy and at delivery. Br J Nutr 2009;101:399-407.

27 Dirix CE, Kester AD, Hornstra G: Associations between term birth dimensions and prenatal exposure to essential and trans fatty acids. Early Hum Dev 2009;85:525-530.

28 Lemaitre RN, King IB, Raghunathan TE, Pearce RM, Weinmann S, Knopp RH, Copass MK, Cobb LA, Siscovick DS: Cell membrane trans-fatty acids and the risk of primary cardiac arrest. Circulation 2002;105: 697-701.

29 Sun Q, Ma J, Campos H, Hankinson SE, Manson JE, Stampfer MJ, Rexrode KM, Willett WC, Hu FB: A prospective study of trans fatty acids in erythrocytes and risk of coronary heart disease. Circulation 2007;115: 1858-1865.

30 L'Abbé MR, Stender S, Skeaff M, Ghafoorunissa, Tavelle M: Approaches to removing trans fats from the food supply in industrialized and developing countries. Eur J Clin Nutr 2009;63:S50-S67.

31 Bouwstra H, Dijck-Brouwer J, Decsi T, Boehm G, Boersma ER, Muskiet FA, Hadders-Algra M: Neurologic condition of healthy term infants at 18 months: positive association with venous umbilical DHA status and negative association with umbilical trans-fatty acids. Pediatr Res 2006;60:334339.

32 Jendryczko A, Gruszczynski J, Tomala J, Szpyrka G: Unsaturated fatty acids of trans isomers in plasma of pregnant women and birth weight (in Polish with English summary). Ginekol Pol 1993;64:113-116.

33 Morrison JA, Glueck CJ, Wang P: Dietary trans fatty acid intake is associated with increased fetal loss. Fertil Steril 2008;90:385390.

34 van Houwelingen AC, Hornstra G: trans fatty acids in early human development; in Galli C, Simopoulos AP, Tremoli E (eds): Fatty Acids and Lipids: Biological Aspects. World Rev Nutr Diet. Basel, Karger, 1994, vol 75, pp 175-178.

35 van Eijsden M, Hornstra G, van der Wal MF, Vrijkotte TG, Bonsel GJ: Maternal n-3, $\mathrm{n}-6$, and trans fatty acid profile early in pregnancy and term birth weight: a prospective cohort study. Am J Clin Nutr 2008; $87: 887-895$. 